from this survey were published in December 2000. A Question of Choice is available from NSF and can be accessed via our website at nsf.org.uk/information /

opinion \& debate research. Nearly two thirds (62\%) of respondents had not been offered a choice of medicine and nearly half $(46 \%)$ had not received written information about sideeffects. The most frequently experienced side-effects were loss of energy (63\%) and weight gain (62\%). Both of these were tolerated by less than $40 \%$ of people.

A consistent finding from the survey was that in the anti-psychotic group atypicals were significantly associated with positive outcomes when compared to typical drugs.

What becomes clear from talking and listening to the views of people with mental illness and their carers is that they want informed choice. They do not want one medicine alone, or one group, they want the widest access possible to find the best fit. It is hoped that the
NICE guidelines will support this pragmatic view and enable all those involved in mental health care to work together to increase people's quality of life.

\section{References}

ADAMS, C. \& NHS CENTRE FOR REVIEWS AND DISSEMINATIONS (1999) Effective Health Care Bulletin, 5, no 6.

HOGMAN, G. (1996) is Cost a Factor? A Survey by the National Schizophrenia Fellowship of the Experiences and Views of Psychiatrists on New Drugs for the Treatment of Schizophrenia. London: NSF.

Gary Hogman Head of Policy and Campaigns, National Schizophrenia Fellowship, 30 Tabernacle Street, London EC2A 4DD
TAYLOR, D. (1999) Is Costa Factor-II? A Survey of Psychiatrists and Health Authorities to Determine the Factors influencing the Prescribing and Funding of Atypical Antipsychotics (eds M. Sharman \& M. Shubhra). London: NSF.

Psychiatric Bulletin (2001), 25, 290-291

\title{
DAVID HEALY
}

\section{Evidence biased psychiatry? ${ }^{\dagger}$}

For a variety of reasons evidence-based medicine is currently in vogue. The evidence most commonly appealed to comes from randomised controlled trials (RCTs), even though the creator of the RCT, Austin Bradford Hill, argued in the 1960s that while it was good to see some swing toward using RCTs, if we ever ended up thinking that RCTs were the only method to evaluate a treatment the pendulum would not only have swung too far, it would have come off its hook (Hill, 1966).

In common with any other scientific experiment, RCTs are designed to test a null hypothesis. That debriefing after trauma, for example, is no better than non-intervention. These evaluative methods work best when they stop therapeutic bandwagons in their tracks. In the case of the first clinical trials with psychotropic agents, the results were such that sceptics could not readily maintain the position that there was no treatment effect with these drugs, however uncertain they might have remained about the merits of treatment in the longer run.

The majority of recent psychotropic drug trials are business rather than scientific exercises, constructed for the purposes of achieving regulatory approval and thereafter market penetration. In the case of regulatory applications for the newer antipsychotics, adopting the null hypothesis for the data leaves fair-minded observers unable to maintain the position that these drugs are without effect. Some recent studies have included comparator arms, using haloperidol in particular. But none of these studies have led to a regulatory labelling of

$\uparrow$ See editorial, pp. 281-282 and pp. 284-286, pp. $287-288$ pp. 289-290 and pp. 291-292 the newer agents as superior to or preferable to haloperidol. In the absence of regulatory indications that the null hypothesis has not been shown to hold when new and older agents are compared, it is difficult to see how the makers of guidelines can make many statements comparing agents. It also becomes possible to see why those who might frame guidelines leave themselves open to a legal challenge from pharmaceutical companies, as has happened in other areas of medicine.

There are many statisticians who doubt the power of even well-designed RCTs to generalise to the real world (Gigerenzer, 1993). Company sponsored RCTs invariably recruit samples of convenience, which by definition do not readily sustain an extrapolation to normal clinical practice. In addition, senior investigators on the trials for some of the newer antipsychotics have been jailed, for reasons that leave considerable uncertainty as to how many of the patients in these trials actually existed and how well they were assessed (Stecklow \& Johannes, 1997). Nevertheless, the weight of trial data makes it all but impossible to maintain the position that these new agents are without effect. There can, however, be considerable uncertainty as to how these effects translate into clinical practice. Epidemiologists with doubts about the generalisability of RCT data would prefer harder end-points, such as return to work or suicide figures from large simple trials. Using RCTs, the extrapolation from treatment effects to treatment efficacy would be more convincing if effects were demonstrated across a range of measurement domains from physician-based disease specific scales, through patientbased disease specific and physician-based global functioning scales to patient-based global functioning or quality of life scales. This has not been demonstrated for either new or older agents. Even if it had been demonstrated and the results for shorter-term efficacy were convincing, there could be doubts about the longer-term effectiveness of treatment, owing to the unexplored impact of withdrawal syndromes (Tranter \& Healy, 1998). 
While few clinicians would doubt that there is some treatment efficacy and effectiveness for both older and newer agents, given that there is a vanishingly small amount of RCT data to establish these points, it must be clear that any attempt to move beyond this state of affairs to proper comparator studies between compounds raises the level of complexity of the evaluative exercise exponentially. It should also be clear that assessments of what treatments have been doing for the past 50 years have depended on clinical judgements, informed by visible factors such as return to work and feedback from patients, rather than judgements informed by clinical trial data. The megadose regimens of antipsychotics used during the 1980s illustrate the scope of factors other than clear benefits to patients to influence perceptions of outcomes. In addition to biases from theoretical preconceptions, a growing company outlay, currently estimated at approximately $\mathrm{f} 8000$ per doctor per annum in Western medical settings (Kirkpatrick, 2000), is unlikely to be without effect on how clinical outcomes are perceived and trial data interpreted.

We please ourselves with notions that a greater use of RCTs has ushered us into an era of evidence-based medicine. In fact, there is every reason to believe that far from stopping therapeutic bandwagons in their tracks, RCTs and the guidelines that stem from them have latterly become the fuel for new bandwagons. What should clinicians and budget holders do in the circumstances? There is little they can do. It might be possible to get a consensus for guidelines stating that high dose antipsychotics are not desirable. But does high dose include the $30 \mathrm{mg}$ and more of olanzapine per day widely used at present or the combination of olanzapine with other antipsychotics in both oral and depot form? And what about the use of these agents for mania and for personality disorders? The government at present chides psychiatrists for shirking their duties in minimising risks to the public from patients with psychoses and personality disorders. Over and above the effects of any of the newer agents on schizophrenia processes, there lies the fact that these agents control behaviours. How will health care purchasers, providers and the government react, if, following the next attack and death in the community, the media latches on to the fact that the perpetrator was maintained on haloperidol $2.5 \mathrm{mg}$ per day?

The current situation indeed, for two reasons, perhaps offers purchasers and patients the best they can hope for. First, companies are openly advocating lower doses of antipsychotics and monotherapy. Second, our ignorance of how these new agents actually mediate their effects means that, temporarily at least, clinical observations of patient benefits are probably more influential than theoretical preconceptions in tailoring appropriate treatments.

\section{References}

GIGERENZER, G. (1993) The Superego, the Ego, and the Id in statistical reasoning. In A Handbook for Data Analysis in the Behavioral Sciences, Vol. 1. Methodological Issues (eds G. Keren \& C. Lewis), pp. 331-339. Hillsdale, NJ: L. Erlbaum.

HILL, A. B. (1966) Reflections on the controlled trial. Annals of the Rheumatic Diseases, 25, 107-113.

KIRKPATRICK, D. D. (2000) Inside the Happiness Business, May 15, pp. 36-43.

David Healy Director of Department of Psychological Medicine, University of Wales College of Medicine, Hergest Unit, Ysbyty Gwynedd, Bangor Gwynedd LL572PW
STECKLOW, S. \& JOHANNES, I. (1997) Questions arise on new drug testing. Drug makers relied on clinical researchers who now await trial. Wall Street Journal, 15 August, pp.1-3.

TRANTER, R. \& HEALY, D. (1998)

Neuroleptic discontinuation Psychopharmacology, 12, 306-311. syndromes. Journal of

Psychiatric Bulletin (2001), 25, 291-292

\section{"Nobody ever expects the Spanish Inquisition" (Python, 1991) $\dagger$}

Guidelines are systematically developed statements designed to help practitioners and patients make decisions about appropriate health care for specific circumstances (Jackson \& Feder, 1998). 'Help' is an important word. Guidelines/guides, in most instances, may suggest a road to take in order to travel from $A$ to $B$, and make explicit why those suggestions have been made. Provision of this information respects the traveller's ability to assimilate the information, and make decisions on applicability. The traveller is then not constrained by information but helped by it. At the end of the day, for clear reasons, a different road may be chosen.

Should guidelines be used unthinkingly to dictate practice, then the worst fears of both those with antipathy to evidence-based medicine (EBM), and those who support EBM are realised. Practitioners hostile to their perceived impressions of evidence-based practice will see inappropriately constructed or implemented guidelines as constraining of clinical freedom, often drawn up by those losing touch with 'real world' medicine and cries of 'dictation by numbers' will be heard throughout the land (Grahame-Smith, 1995). On the other hand, the accusation of 'dictation by numbers' - justified if guidelines are used as stipulations for practice - will also disturb those who wished EBM to be the "conscientious, explicit and judicious use of current best evidence in making decisions about the care of individual patients" (Sackett et al, 1996).

Readers of guidelines should make up their own minds whether guidelines "made explicit identification of

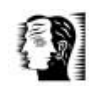

opinion \& debate 